\title{
ON THE OPTIMAL ASYMPTOTIC EIGENVALUE BEHAVIOR OF WEAKLY SINGULAR INTEGRAL OPERATORS
}

\author{
FERNANDO COBOS, SVANTE JANSON, AND THOMAS KÜHN
}

(Communicated by Palle E. T. Jorgensen)

\begin{abstract}
We improve the known results on eigenvalue distributions of weakly singular integral operators having (power) order of the singularity equal to half of the dimension of the underlying domain. Moreover we show that our results are the best possible.
\end{abstract}

\section{INTRODUCTION}

Let $\Omega$ be a bounded domain in $\mathbb{R}^{N}$. For $0<\alpha<1$ and $\gamma \in \mathbb{R}$ or $\alpha=0$ and $\gamma<-1$, let us consider the weakly singular kernel

$$
K(x, y)=\frac{L(x, y)(1+|\log \|x-y\||)^{\gamma}}{\|x-y\|^{N(1-\alpha)}}, \quad x, y \in \Omega,
$$

where $L \in L_{\infty}\left(\Omega^{2}\right)$ and $\|\cdot\|$ stands for the Euclidean norm in $\mathbb{R}^{N}$. We denote by $T_{K}=T_{L, \alpha, \gamma}$ the integral operator defined by $K$, that is,

$$
T_{K} f(x)=T_{L, \alpha, \gamma} f(x)=\int_{\Omega} K(x, y) f(y) d y .
$$

This operator class contains the integral operators originating as inverses of many differential operators.

The operator $T_{K}$ is compact in the Hilbert space $L_{2}(\Omega)$. Thus we can arrange the eigenvalues of $T_{K}$ in a sequence $\left(\lambda_{n}\left(T_{K}\right)\right)$, where each eigenvalue is repeated as many times as it multiplicity indicates and they are in nonincreasing order of modulus,

$$
\left|\lambda_{1}\left(T_{K}\right)\right| \geq\left|\lambda_{2}\left(T_{K}\right)\right| \geq \cdots \geq 0 .
$$

If $T_{K}$ has less than $n$ eigenvalues, then we put

$$
\lambda_{n}\left(T_{K}\right)=\lambda_{n+1}\left(T_{K}\right)=\cdots=0 .
$$

Received by the editors June 1, 1990 .

1980 Mathematics Subject Classification (1985 Revision). Primary 47G05, 47B10.

Key words and phrases. Integral operators, eigenvalues, singular numbers.

The research of the first author was partially supported by the "Civilingenjör Gustaf Sigurd Magnusons fond för främjande av vetenskapen inom ämnet matematik" of the Royal Swedish Academy of Sciences (Kungl. Vetenskapsakademien) while he was at the University of Stockholm. 
The compactness of $T_{K}$ implies that $\left(\lambda_{n}\left(T_{K}\right)\right)$ converges to zero. The order of decay of the eigenvalues is a problem that has attracted the attention of a number of authors. Let us mention, for example, the papers by Kostometov [8], König, Retherford, and Tomczak-Jaegermann [7], König [5], Carl and Kühn [1], and Cobos and Kühn [2, 3]. It turned out that the eigenvalues $\left(\lambda_{n}\left(T_{L, \alpha, \gamma}\right)\right)$ decay as $O\left(M_{\alpha, \gamma}(n)\right)$ where

$$
M_{\alpha, \gamma}(n)= \begin{cases}(\log n)^{\gamma+1} & \text { if } \alpha=0, \gamma<-1, \\ n^{-\alpha}(\log n)^{\gamma} & \text { if } 0<\alpha<1 / 2, \gamma \in \mathbb{R}, \\ n^{-1 / 2}(\log n)^{\gamma+1 / 2} & \text { if } \alpha=1 / 2, \gamma>-1 / 2, \\ n^{-1 / 2}(\log \log n)^{1 / 2} & \text { if } \alpha=1 / 2, \gamma=-1 / 2,\end{cases}
$$

and

$$
\left(\lambda_{n}\left(T_{L, \alpha, \gamma}\right)\right) \in l_{2} \text { if } 1 / 2<\alpha<1, \gamma \in \mathbb{R} \text { or } \alpha=1 / 2, \gamma<-1 / 2 .
$$

Except for the case $\alpha=1 / 2$ and $\gamma \geq-1 / 2$, all these estimates are the best possible. By this we mean that there exist weakly singular integral kernels $K=$ $K_{L, \alpha, \gamma}$ of the type $(*)$ such that

$$
\left|\lambda_{n}\left(T_{L, \alpha, \gamma}\right)\right| \simeq M_{\alpha, \gamma}(n)
$$

(that is, for such a kernel one also has $M_{\alpha, \gamma}(n)=O\left(\left|\lambda_{n}\left(T_{L, \alpha, \gamma}\right)\right|\right)$ ). See [3, Theorem 5].

But if $\alpha=1 / 2$ and $\gamma \geq-1 / 2$, the optimality of the estimate

$$
\left|\lambda_{n}\left(T_{L, 1 / 2, \gamma}\right)\right|=O\left(M_{1 / 2, \gamma}(n)\right), \quad \gamma \geq-1 / 2,
$$

is only known in a weaker sense. By means of random techniques one can show that there is a sequence of positive integers $(j(n))_{n \in \mathbb{N}}$ tending to $\infty$ with $n$ such that

$$
\sup _{\|L\|_{\infty} \leq 1} \mid \lambda_{j(n)}\left(T_{L, 1 / 2, \gamma}\right) \simeq M_{1 / 2, \gamma}(j(n)) .
$$

See $[5,6,3]$.

Hence there is still some room for improving $(* *)$.

In this paper we show that in fact

$$
\sum_{j=1}^{n}\left|\lambda_{j}\left(T_{L, 1 / 2, \gamma}\right)\right|^{2}= \begin{cases}O(\log \log n) & \text { if } \gamma=-1 / 2 \\ O\left((\log n)^{2 \gamma+1}\right) & \text { if } \gamma>-1 / 2\end{cases}
$$

holds. This clearly improves $(* *)$ since

$$
\left|\lambda_{n}\left(T_{L, 1 / 2, \gamma}\right)\right| \leq n^{-1 / 2}\left(\sum_{j=1}^{n}\left|\lambda_{j}\left(T_{L, 1 / 2 . \gamma}\right)\right|^{2}\right)^{1 / 2} .
$$

Moreover, we give examples of weakly singular integral operators of the type $\alpha=1 / 2, \gamma \geq-1 / 2$, having precisely this order of decay of the eigenvalues. Therefore, the estimate $(* * *)$ is the best possible. 


\section{ESTIMATES FOR EIGENVALUES}

We shall use the approach developed in [3]. In particular, we shall need the singular numbers $\left(s_{n}\left(T_{K}\right)\right)$ of $T_{K}$, which are defined as the eigenvalues of $\left[T_{K}^{*} T_{K}\right]^{1 / 2}$,

$$
s_{n}\left(T_{K}\right)=\lambda_{n}\left(\left[T_{K}^{*} T_{K}\right]^{1 / 2}\right), \quad n=1,2, \ldots .
$$

We shall also use the fact that the integral operator associated with a square integrable kernel $K$ is a Hilbert-Schmidt operator and the following holds:

$$
\sum_{j=1}^{\infty} s_{j}\left(T_{K}\right)^{2}=\int_{\Omega} \int_{\Omega}|K(x, y)|^{2} d x d y
$$

(see for example, the books by Gohberg and Krein [4] or by König [6]).

We first estimate the singular numbers of integral operators defined by kernels $K: \Omega^{2} \rightarrow \mathbb{C}$ of the form

$$
K(x, y)=L(x, y) f\left(\|x-y\|^{N}\right), \quad x, y \in \Omega,
$$

where $L \in L_{\infty}\left(\Omega^{2}\right)$ and $f \in L_{1}([0, \infty))$ is a nonnegative function such that for every positive real number $a$ the function $f$ belongs to $L_{2}([a, \infty))$.

Lemma 1. Let $\Omega \subset \mathbb{R}^{N}$ be a bounded domain and let $K$ be a kernel of the form $(\Delta)$. Then, for every $a>0$ and each $n \in \mathbb{N}$, we have

$$
\sum_{j=1}^{n} s_{j}\left(T_{K}\right)^{2} \leq c\|L\|_{\infty}^{2}\left[n\left(\int_{0}^{a} f(t) d t\right)^{2}+\int_{a}^{\infty} f(t)^{2} d t\right],
$$

where the constant $c$ only depends on $\Omega$.

Proof. Split the kernel $K$ as $K_{1}+K_{2}$ where

$$
K_{1}(x, y)= \begin{cases}K(x, y) & \text { if }\|x-y\|^{N} \leq a \\ 0 & \text { elsewhere }\end{cases}
$$

and

$$
K_{2}(x, y)=K(x, y)-K_{1}(x, y) .
$$

One can easily check by Schur's lemma, that the norm of $T_{K_{1}}$ satisfies

$$
\left\|T_{K_{1}}\right\| \leq \sigma_{N}\|L\|_{\infty} \int_{0}^{a} f(t) d t
$$

where $\sigma_{N}$ is the volume of the $N$-dimensional Euclidean unit ball.

The other integral operator is a Hilbert-Schmidt operator because $K_{2}$ is square integrable. Thus

$$
\begin{aligned}
\sum_{j=1}^{\infty} s_{j}\left(T_{K_{2}}\right)^{2} & =\int_{\Omega} \int_{\Omega}\left|K_{2}(x, y)\right|^{2} d x d y \\
& \leq\|L\|_{\infty}^{2} \iint_{\substack{\|x-y\|^{N}>a \\
x, y \in \Omega}} f\left(\|x-y\|^{N}\right)^{2} d x d y \\
& \leq \operatorname{vol}(\Omega) \sigma_{N}\|L\|_{\infty}^{2} \int_{a}^{\infty} f(t)^{2} d t
\end{aligned}
$$


Consequently, using that

$$
s_{j}\left(T_{K}\right) \leq\left\|T_{K_{1}}\right\|+s_{j}\left(T_{K_{2}}\right), \quad j=1,2, \ldots,
$$

we obtain

$$
\begin{aligned}
\sum_{j=1}^{n} s_{j}\left(T_{K}\right)^{2} & \leq \sum_{j=1}^{n}\left(\left\|T_{K_{1}}\right\|+s_{j}\left(T_{K_{2}}\right)\right)^{2} \\
& \leq 2\|L\|_{\infty}^{2}\left(\sigma_{N}^{2}+\sigma_{N} \operatorname{vol}(\Omega)\right)\left[n\left(\int_{0}^{a} f(t) d t\right)^{2}+\int_{a}^{\infty} f(t)^{2} d t\right] .
\end{aligned}
$$

Now we are in a position to establish

Theorem 2. Let $\Omega \subset \mathbb{R}^{N}$ be a bounded domain and let $K: \Omega^{2} \rightarrow \mathbb{C}$ be a kernel of the form $(*)$ with $\alpha=1 / 2$ and $\gamma \geq-1 / 2$. Then $\left(\sum_{j=1}^{n} s_{j}\left(T_{K}\right)^{2}\right)$ and $\left(\sum_{j=1}^{n}\left|\lambda_{j}\left(T_{K}\right)\right|^{2}\right)$ are of asymptotic order $O\left(J_{\gamma}(n)\right)$, where

$$
J_{\gamma}(n)= \begin{cases}\log \log n & \text { if } \gamma=-1 / 2 \\ (\log n)^{2 \gamma+1} & \text { if } \gamma>-1 / 2\end{cases}
$$

Proof. According to Weyl's inequality, we have

$$
\sum_{j=1}^{n}\left|\lambda_{j}\left(T_{K}\right)\right|^{2} \leq \sum_{j=1}^{n} s_{j}\left(T_{K}\right)^{2}, \quad n=1,2, \ldots,
$$

hence it suffices to prove the estimate for the singular numbers. Put

$$
f(t)= \begin{cases}\left(1+\frac{1}{N}|\log t|\right)^{\gamma} / t^{1 / 2} & \text { if } 0<t<(\operatorname{diam}(\Omega))^{N}, \\ 0 & \text { elsewhere. }\end{cases}
$$

It is not hard to check that

$$
\left(\int_{0}^{1 / n} f(t) d t\right)^{2} \simeq(\log n)^{2 \gamma} / n
$$

and

$$
\int_{1 / n}^{\infty} f(t)^{2} d t \simeq \begin{cases}\log \log n & \text { if } \gamma=-1 / 2 \\ (\log n)^{2 \gamma+1} & \text { if } \gamma>-1 / 2\end{cases}
$$

Hence the desired estimate follows from Lemma 1.

We finish this note with an example of a weakly singular integral operator on $\Omega=[0,1]$ showing the previous estimates are the best possible.

Theorem 3. Let $\gamma \geq-1 / 2$ and let

$$
K(x, y)=\left\{\begin{array}{ll}
(1-\log |x-y|)^{\gamma} /|x-y|^{1 / 2} & \text { if }|x-y| \leq 1 / 2, \\
0 & \text { if }|x-y|>1 / 2,
\end{array} \quad x, y \in[0,1] .\right.
$$

Then the eigenvalues of the weakly singular integral operator $T_{K}$ satisfy

$$
\sum_{j=1}^{n}\left|\lambda_{j}\left(T_{K}\right)\right|^{2} \simeq J_{\gamma}(n)
$$


Proof. First note that the operator $T_{K}$ is selfadjoint because the kernel $K(x, y)$ is real valued and symmetric. Then it follows from the spectral representation theorem that

$$
\left|\lambda_{n}\left(T_{K}\right)\right|=s_{n}\left(T_{K}\right), \quad n=1,2, \ldots
$$

Thus, we only need to show that

$$
J_{\gamma}(n)=O\left(\sum_{j=1}^{n} s_{j}\left(T_{K}\right)^{2}\right) .
$$

With this aim, put

$$
f(t)=(1-\log |t|)^{\gamma} /|t|^{1 / 2}, \quad|t| \leq 1 / 2,
$$

write $\tilde{f}$ for its 1-periodic extension to $\mathbb{R}$, and denote by $C_{f}$ the integral operator on $L_{2}([0,1])$ defined by the kernel

$$
K(x, y)=\tilde{f}(x-y) .
$$

The operator $C_{f}$ is selfadjoint, as well. Hence

$$
s_{n}\left(C_{f}\right)=\left|\lambda_{n}\left(C_{f}\right)\right|, \quad n=1,2, \ldots
$$

One can easily verify that the sequence of the eigenvalues of $C_{f}$ is precisely the nonincreasing rearrangement $\left(\hat{f}(n)^{*}\right)_{n \in \mathbb{N}}$ of the Fourier coefficients $(\hat{f}(j))_{j \in \mathbb{Z}}$ of $f$, and for them we know (see [3, Lemma 4]) that

$$
\hat{f}(n)^{*} \geq c(\log n)^{\gamma} / n^{1 / 2}, \quad n=2,3, \ldots,
$$

where the constant $c$ depends only on $\gamma$. Thus,

$$
c^{2}(\log n)^{2 \gamma} / n \leq s_{n}\left(C_{f}\right)^{2}, \quad n=2,3, \ldots
$$

On the other hand, the operator $C_{f}-T_{K}$ is a Hilbert-Schmidt operator since its kernel is square integrable. Hence we have

$$
\sum_{j=1}^{\infty} s_{j}\left(C_{f}-T_{K}\right)^{2}<\infty .
$$

Taking into account that singular numbers satisfy

$$
s_{2 j}\left(C_{f}\right) \leq s_{j}\left(C_{f}-T_{K}\right)+s_{j}\left(T_{K}\right), \quad j=1,2, \ldots,
$$


we obtain

$$
\begin{aligned}
J_{\gamma}(n) & \simeq \sum_{j=2}^{n}(\log j)^{2 \gamma} / j \simeq \sum_{j=1}^{n} c^{2}(\log 2 j)^{2 \gamma} / 2 j \\
& \leq \sum_{j=1}^{n} s_{2 j}\left(C_{f}\right)^{2} \leq \sum_{j=1}^{n}\left[s_{j}\left(C_{f}-T_{K}\right)+s_{j}\left(T_{K}\right)\right]^{2} \\
& \leq 2\left[\sum_{j=1}^{n} s_{j}\left(C_{f}-T_{K}\right)^{2}+\sum_{j=1}^{n} s_{j}\left(T_{K}\right)^{2}\right] \\
& \leq 2\left[c_{1}+\sum_{j=1}^{n} s_{j}\left(T_{K}\right)^{2}\right] .
\end{aligned}
$$

This gives $(\Delta \Delta)$ and completes the proof.

\section{REFERENCES}

1. B. Carl and T. Kühn, Entropy and eigenvalues of certain integral operators, Math. Ann. 268 (1984), 127-136.

2. F. Cobos and T. Kühn, Entropy and eigenvalues of weakly singular integral operators, Integral Equations and Operator Theory 11 (1988), 64-86.

3. __ Eigenvalues of weakly singular integral operators, J. London Math. Soc. (2) 41 (1990), 323-335.

4. I. C. Gohberg and M. G. Krein, Introduction to the theory of linear non-selfadjoint operators, Amer. Math. Soc., Providence, RI, 1969.

5. H. König, Some remarks on weakly singular integral operators, Integral Equations and Operator Theory 3 (1980), 397-407.

6. __ Eigenvalue distribution of compact operators, Birkhäuser, Basel, Boston, MA, and Stuttgart, 1986.

7. H. König, J. R. Retherford, and N. Tomczak-Jaegermann, On the eigenvalues of $(p, 2)$ summing operators and constants associated to normed spaces, J. Funct. Anal. 37 (1980), $88-126$.

8. G. P. Kostometov, Asymptotic behaviour of the spectrum of integral operators with a singularity on the diagonal, Math. USSR-Sb. 23 (1974), 417-424.

Departamento de Matemáticas, Facultad de Ciencias, Universidad Autónoma de MADRID, 28049 MADRID, SPAIN

Department of Mathematics, Uppsala University, Thunbergsvägen 3, S-75238 UpPSALA, SWEDEN

Sektion Mathematik, Universität LeipZig, D-7010 Leipzig, Germany 